\title{
SISTEMAS DE CULTIVO E MANEJO DE ÁGUA NA PRODUÇÃO DE DIFERENTES GENÓTIPOS DE ARROZ EM VÁRZEA, NO ESTADO DE RORAIMA ${ }^{1}$
}

\author{
Antonio Carlos Centeno Cordeiro ${ }^{2}$, Elias Suhre ${ }^{3}$, Roberto Dantas de Medeiros ${ }^{2}$, Aloisio Alcantara Vilarinho ${ }^{2}$
}

\section{ABSTRACT \\ CROPPING SYSTEMS AND WATER MANAGEMENT \\ OF DIFFERENT LOWLAND RICE GENOTYPES YIELD, RORAIMA STATE, BRAZIL}

This study had the objective of assessing test lines and rice cultivars, in relation to grain yield and other agronomic traits, under different cropping and irrigation management systems, in the Rio Branco lowlands, municipality of Cantá, Roraima State, Brazil. A randomized complete block design was used, with four replications. Nineteen test lines and six cultivars were assessed. Significant differences for most of the evaluated traits were observed. The highest grain yield was obtained under a cropping system with continuous flooding irrigation and row sowing $\left(8,684 \mathrm{~kg} \mathrm{ha}^{-1}\right)$ and the lowest ones were obtained under cropping systems with intermittent irrigation, independently of sowing method. The control cultivars IRGA417, BRS Jaçanã, BRS Jaburu, and Roraima presented a good performance, under different cropping systems. The test lines BRA 051250, BRA 051126, CNA 10900, BRA 051135, and CNA 110114 are promising for future cultivar releases to the local production systems.

KEY-WORDS: Oryza sativa; genotype-environment interaction; grain yield.

\section{INTRODUÇÃO}

O arroz (Oryza sativa L.) é um dos cereais de maior importância social e econômica para o mundo. É responsável pela alimentação de dois terços da população mundial e fornece, aproximadamente, $20 \%$ da energia e $15 \%$ da proteína necessária ao ser humano. O continente asiático é o maior produtor e consumidor mundial. O Brasil ocupa a nona posição, entre os principais países produtores, sendo o Rio Grande do Sul o Estado com a maior produção no País, respondendo por mais da metade desta produção (Azambuja et al. 2004, Duarte 2006).

\section{RESUMO}

O presente trabalho teve como objetivo avaliar linhagens experimentais e cultivares de arroz, com relação à produtividade de grãos e outras características agronômicas, em diferentes sistemas de cultivo e manejo de água, em várzea do Rio Branco, município de Cantá, Estado de Roraima. O delineamento experimental utilizado foi o de blocos casualizados, com quatro repetições. Dezenove linhagens experimentais e seis cultivares foram avaliadas. Detectaram-se diferenças significativas, para a maioria das características. A maior produtividade de grãos foi obtida no sistema de cultivo com irrigação por inundação contínua e semeadura em linhas $\left(8.684 \mathrm{~kg} \mathrm{ha}^{-1}\right)$ e as menores nos sistemas com irrigação intermitente, independentemente do método de semeadura. As cultivares testemunhas IRGA 417, BRS Jaçanã, BRS Jaburu e Roraima apresentaram bom desempenho produtivo, nos diferentes sistemas avaliados. As linhagens experimentais BRA 051250, BRA 051126, CNA 10900, BRA 051135 e CNA 110114 são promissoras para futuras liberações de cultivares aos sistemas de produção local.

PALAVRAS-CHAVE: Oryza sativa; interação genótipo x ambiente; rendimento de grãos.

No Estado de Roraima, o agronegócio do arroz tem participação significativa na geração de emprego, renda e no Produto Interno Bruto (PIB), sendo uma das poucas cadeias produtivas efetivamente estabilizadas no Estado (Cordeiro et al. 2007). Aárea semeada no Estado tem crescido anualmente e com potencial de expansão, tendo em vista a existência de 160.211 hectares de solos de várzeas, mapeados em região de savana. Neste universo, 124.888 hectares correspondem a solos com aptidão agrícola para o cultivo do arroz irrigado (Provam 1996).

Atualmente, os principais sistemas de cultivo de arroz em várzea, utilizados pelos produtores

1. Trabalho recebido em set./2009 e aceito para publicação em set./2010 (n registro: PAT 7520/ DOI: 10.5216/pat.v40i3.7520).

2. Embrapa Roraima, Boa Vista, RR, Brasil.E-mails: acarlos@cpafrr.embrapa.br, roberto@cpafrr.embrapa.br, aloisio@cpafrr.embrapa.br.

3. Ministério Público Federal, Procuradoria Geral da República, Assessoria Pericial, Brasília, DF, Brasil.

E-mail: eliassuhre@yahoo.com.br. 
locais, são a irrigação por inundação contínua, com semeadura a lanço (principal) ou em linhas, e o sistema de várzeas úmidas, sendo, este último, somente utilizado no período chuvoso. Segundo Rangel (1990), os sistemas de cultivo de arroz irrigado e de várzea úmida, apesar de suas peculiaridades, possuem pontos de convergência, sendo o principal deles a quase inexistência de estresse hídrico. Assim, uma das possibilidades para se reduzir o custo de produção seria a seleção de linhagens com alto potencial produtivo, para estas duas condições.

Quanto ao manejo de água, embora a cultura do arroz possa ser irrigada por qualquer método de irrigação, o sistema por inundação contínua é o mais utilizado, pois apresenta maior praticidade na execução, com consequente aumento na produtividade, melhoria na qualidade industrial de grãos e favorecimento no controle de plantas daninhas (Medeiros et al. 1997). Apesar disso, Medeiros et al. (1995) e Cordeiro et al. (2004) encontraram produtividades semelhantes, utilizando irrigação contínua e intermitente por inundação, e Cordeiro (2005a), em ensaio de Valor de Cultivo e Uso (VCU), obteve, em média, maior produção, em sistema de irrigação intermitente.

Apesar da existência de água de qualidade e em abundância em Roraima, a orizicultura é uma importante consumidora deste recurso natural. Com os principais cultivos situados às margens do Rio Branco e seus afluentes, à montante do centro consumidor de Boa Vista (capital do Estado), a busca por sistemas de cultivo e cultivares mais produtivas e eficientes no uso da água são relevantes para a sustentabilidade desta atividade. Neste sentido, os ensaios de VCU são de fundamental importância para a seleção de cultivares melhor adaptadas aos ecossistemas de várzea do Estado, com alto potencial produtivo e que contribuam para o uso racional da água e aumento da oferta deste alimento básico na dieta dos brasileiros, a um preço mais acessível.

O objetivo do presente trabalho foi avaliar vinte e cinco genótipos de arroz, entre linhagens experimentais e cultivares, com relação à produtividade de grãos e outras características agronômicas, em diferentes sistemas de cultivo e de manejo da água de irrigação, em várzea do Rio Branco, município de Cantá, Estado de Roraima.

\section{MATERIAL E MÉTODOS}

Foram conduzidos quatro experimentos, oriundos do programa de melhoramento de arroz da Embrapa Roraima, designados como Ensaios de Valor de Cultivo e Uso (VCU), em área de várzea do Rio Branco, no município de Cantá, RR (248'29”N, 60 39'19'W e $61 \mathrm{~m}$ de altitude). O clima da região é classificado por Köppen como Awi, com precipitação média anual de $1.600 \mathrm{~mm}$. Os meses mais secos estão entre dezembro e março, com apenas $10 \%$ da precipitação anual. A vegetação primária ocorrente na região é de savana.

Os experimentos foram conduzidos no período de dezembro de 2007 e abril de 2008, em solo classificado como Gleissolo Háplico Tb distrófico (Embrapa 2006). Os resultados das análises química e granulométrica das amostras de solo, na camada 0,0-0,2 m de profundidade, revelaram as seguintes características: $\mathrm{pH}=5,2 ; \mathrm{MO}=11,9 \mathrm{~g} \mathrm{~kg}^{-1} ; \mathrm{P}=2,16 \mathrm{mg} \mathrm{dm}^{-3}$; $\mathrm{K}=0,07 \mathrm{cmol}_{\mathrm{c}} \mathrm{dm}^{-3} ; \mathrm{Ca}=0,64 \mathrm{cmol}_{\mathrm{c}} \mathrm{dm}^{-3} ; \mathrm{Mg}=$ $0,28 \mathrm{cmol}_{\mathrm{c}} \mathrm{dm}^{-3} ; \mathrm{Al}=1,83 \mathrm{cmol}_{\mathrm{c}} \mathrm{dm}^{-3} ;$ argila $=43 \%$; silte $=50 \%$; e areia $=7 \%$. Estas análises foram realizadas de acordo com os métodos preconizados pela Embrapa (1997).

Cada ensaio foi composto por 19 linhagens experimentais e seis cultivares de arroz, sendo quatro delas consideradas testemunhas (Tabela 1). Os quatro ambientes experimentais, em área de várzea úmida, consistiram de diferentes sistemas de cultivo e de manejo da água: S1 - irrigação por inundação contínua e semeadura em linhas espaçadas de $0,30 \mathrm{~m}$, com densidade de cem sementes viáveis por metro, equivalente a $100 \mathrm{~kg} \mathrm{ha}^{-1} ; \mathrm{S} 2$ - irrigação por inundação intermitente (várzea úmida) e semeadura à semelhança de S1; S3 - irrigação por inundação contínua e semeadura a lanço, na densidade de $100 \mathrm{~kg} \mathrm{ha}^{-1}$ de sementes viáveis; e S4 - irrigação por inundação intermitente e semeadura à semelhança de S3. Cada ensaio foi conduzido em delineamento experimental de blocos ao acaso, com quatro repetições. A dimensão das parcelas foi de $1,8 \mathrm{~m}$ x 5,0 m, com área útil de $1,2 \mathrm{~m} \mathrm{x} \mathrm{4,0} \mathrm{m.}$

O preparo do solo foi realizado com o solo seco e consistiu de uma aração com grade aradora, duas gradagens niveladoras e construção de taipas. A adubação de base foi de $450 \mathrm{~kg} \mathrm{ha}^{-1}$ da fórmula 04-28-20 + Zn. Na semeadura a lanço, as sementes foram incorporadas ao solo com auxílio de grade niveladora e, em seguida, passou-se o 
Tabela 1. Relação dos genótipos de arroz avaliados em ensaios de Valor de Cultivo e Uso (VCU), no Estado de Roraima, entre dezembro de 2007 e abril de 2008, com as respectivas procedências ou cruzamentos de origem.

\begin{tabular}{ccc}
\hline Tratamento Linhagem/Variedade & Origem ou Cruzamento \\
\hline 1 & BRA 051067 & Formoso x IRGA 97-05 \\
2 & BRA 051077 & IRGA 417 x BRS Jaburu \\
3 & BRA 051083 & IRGA 97-05 x CNAi 9020 \\
4 & BRA 051103 & Formoso x IRGA 97-28 \\
5 & BRA 051107 & Formoso x IRGA 97-28 \\
6 & BRA 051108 & IR 22 x CNA 8502 \\
7 & BRA 051125 & IRGA 417 x BRS Jaburu \\
8 & BRA 051126 & IRGA 417 x BRS Jaburu \\
9 & BRA 051129 & IRGA 417 x BRS Jaburu \\
10 & BRA 051130 & IRGA 417 x BRS Jaburu \\
11 & BRA 051134 & IRGA 417 x BRS Jaburu \\
12 & BRA 051135 & IRGA 417 x BRS Jaburu \\
13 & BRA 051179 & BRS TAIM x CNAi 9050 \\
14 & BRA 051250 & Formoso x CNA 8502 \\
15 & BRA 051252 & Formoso x CNA 8319 \\
16 & BRA 041049 & - \\
17 & CNA 10892 & Formoso x CNAi 9022 x Formoso \\
18 & CNA 10900 & Formoso x orizica LLanos 5 x Formoso \\
19 & CNA 11014 & Introdução do CIAT \\
20 & BR IRGA 409 & Testemunha local \\
21 & BRS Jaçanã & CNA6181 x IRI344 x BR IRGA 409 \\
22 & Metica 1 & Testemunha regional \\
23 & Roraima & Testemunha local \\
24 & BRS Jaburu & PDR x P3790F x P5746 \\
25 & IRGA 417 & Testemunha local \\
\hline
\end{tabular}

rolo compactador, para melhorar o contato da semente com o solo. Na semeadura em linhas, as operações de abertura de sulcos, distribuição das sementes e fechamento dos sulcos foram realizadas manualmente. A semeadura foi realizada em $15 / 12 / 2007$, com o solo drenado, e a emergência das plântulas ocorreu em 20/12/2007. A adubação em cobertura foi de $150 \mathrm{~kg} \mathrm{ha}^{-1}$ de ureia $(45 \%$ de $\mathrm{N})$, aplicados em parcelados, sendo $1 / 2$ no início do perfilhamento (15 dias após a emergência) e 1/2 na diferenciação floral (45 dias após a emergência). $\mathrm{O}$ controle de plantas daninhas foi realizado em pré-emergência, após a semeadura, aplicando-se o equivalente a $1,0 \mathrm{~kg}$ de i.a. ha ${ }^{-1}$ de oxadiazon, na forma de pulverização sobre o solo, com umidade próxima à saturação.

Os quatro experimentos foram irrigados através de banhos intermitentes, no período compreendido entre a semeadura e o perfilhamento. A partir deste estágio, foram aplicados os manejos de água (irrigação por inundação intermitente ou irrigação por inundação contínua), conforme cada ambiente experimental. Os sistemas de cultivo com irrigação por inundação contínua foram mantidos com lâmina de água variando de $5 \mathrm{~cm}$ a $15 \mathrm{~cm}$ de altura, controlada diariamente. Nos sistemas de inundação intermitente, a irrigação ocorreu, em média, a cada quatro dias, mantendo-se o solo sempre entre a saturação e a capacidade de campo.
A coleta de dados referentes à floração média (50\%), ciclo (dias até a maturação completa), altura de planta $(\mathrm{cm})$, acamamento (\%), notas de doenças e produtividade de grãos $\left(\mathrm{kg} \mathrm{ha}^{-1}\right)$ foi realizada conforme metodologia preconizada pelo International Rice Research Institute (1996).

Foram realizadas análises de variância individuais e conjuntas, considerando-se cada sistema como um ambiente de teste, e, para a comparação das estimativas das médias dos tratamentos, aplicou-se o teste de Scott \& Knott, em nível de 5\% de probabilidade. Para isto, utilizou-se o aplicativo computacional Sisvar (Ferreira 2008). O teste de homocedasticidade dos quadrados médios dos resíduos, proposto por Gomes (1990), foi aplicado, indicando a possibilidade de realização das análises conjuntas. Foram estimados, ainda, os coeficientes de correlação de Pearson (r), entre as características avaliadas, conforme preconiza Zimmermann (2004).

\section{RESULTADOS E DISCUSSÃO}

Os resumos das análises de variância conjuntas, para as características altura de planta, ciclo, produtividade de grãos, brusone na folha e mancha de grãos, referentes aos quatro sistemas de cultivo, são apresentados na Tabela 2. Devido à alta correlação existente entre floração e ciclo, são apresentados apenas os dados referentes à segunda característica. Quanto à característica acamamento, não foi verificada a sua ocorrência em quaisquer dos tratamentos.

Verifica-se que, de modo geral, ocorreram diferenças significativas $(\mathrm{p}<0,01)$ para todas as características avaliadas. Por outro lado, para as fontes de variação "genótipos" (linhagens experimentais e cultivares) e "genótipos x sistemas", houve diferenças significativas para as características altura de planta, ciclo e produtividade de grãos, indicando comportamento diferenciado dos genótipos para estas características, quando foram avaliados nos quatro sistemas de cultivo (Tabela 2).

Devido à ocorrência da interação genótipos $\mathrm{x}$ sistemas, foi realizada a decomposição da fonte de variação genótipos dentro de sistemas, visando à apresentação e comparação dos resultados das linhagens, para todas as características e por sistema de cultivo. À exceção de brusone na folha e mancha de grãos, bem como altura de planta para genótipos/ $\mathrm{S} 2$, as demais características apresentaram diferenças altamente significativas $(\mathrm{p}<0,01)$, evidenciando am- 
Tabela 2. Análises de variância conjuntas, para as características altura de planta $(\mathrm{cm})$, ciclo (dias até a maturação completa), produtividade de grãos $\left(\mathrm{kg} \mathrm{ha}^{-1}\right)$, brusone na folha $(\mathrm{BF})^{1}$ e mancha de grãos $(\mathrm{MG})^{1}$, em genótipos de arroz cultivados sob quatro sistemas de cultivo e manejo de água na irrigação, em várzea do Rio Branco (Cantá, RR, safra 2007/2008).

\begin{tabular}{lcccrrr}
\hline \multirow{2}{*}{ FV } & \multirow{2}{*}{ GL } & \multicolumn{3}{c}{ Quadrados médios } & \multicolumn{1}{c}{ BF } \\
\cline { 3 - 7 } & & Altura & Ciclo & Produtividade & MG \\
\hline Bloco/Sistema & 12 & $136,90^{* *}$ & $40,49^{* *}$ & $2674478,86^{* *}$ & $5,95^{* *}$ & $3,78^{* *}$ \\
Sistemas (S) & 3 & $3492,72^{* *}$ & $569,81^{* *}$ & $33288510,00^{* *}$ & $23,88^{* *}$ & $22,59^{* *}$ \\
Genótipos (G) & 24 & $202,22^{* *}$ & $78,76^{* *}$ & $2239014,39^{* *}$ & $1,38^{\mathrm{ns}}$ & $1,09^{\mathrm{ns}}$ \\
Interação G X S & 72 & $22,87^{\text {ns }}$ & $14,69^{* *}$ & $1438613,44^{* *}$ & $1,45^{\mathrm{ns}}$ & $1,60^{\mathrm{ns}}$ \\
\hline Genótipos/S & 96 & $67,71^{* *}$ & $30,70^{* *}$ & $1638713,68^{* *}$ & $1,41^{\mathrm{ns}}$ & $1,47^{\mathrm{ns}}$ \\
Genótipos/S1 & 24 & $51,98^{* *}$ & $17,04^{* *}$ & $1644477,53^{* *}$ & $1,18^{\mathrm{ns}}$ & $1,19^{\mathrm{ns}}$ \\
Genótipos/S2 & 24 & $34,26^{\mathrm{ns}}$ & $36,23^{* *}$ & $1252532,58^{* *}$ & $1,58^{\mathrm{ns}}$ & $1,42^{\mathrm{ns}}$ \\
Genótipos/S3 & 24 & $80,98^{* *}$ & $36,92^{* *}$ & $1095298,71^{* *}$ & $1,96^{\mathrm{ns}}$ & $1,46^{\mathrm{ns}}$ \\
Genótipos/S4 & 24 & $103,62^{* *}$ & $32,64^{* *}$ & $2562545,89^{* *}$ & $0,94^{\mathrm{ns}}$ & $1,83^{\mathrm{ns}}$ \\
\hline Erro Médio & 288 & 20,36 & 6,51 & 496010,78 & 1,2 & 1,74 \\
Total & 399 & - & - & - & - & - \\
\hline
\end{tabular}

" $\mathrm{e}^{* *}$ : Valores significativos, respectivamente, aos níveis de $5 \%$ e $1 \%$ de probabilidade, pelo teste $\mathrm{F}$.

${ }^{\text {ns }}$ Valores não significativos, pelo mesmo teste, em nível de $5 \%$ de probabilidade.

${ }^{1}$ BF e MG: Valores expressos em escala de notas visuais (1 a 9), sendo 1 = ausência de lesão e 9 = mais de $75 \%$ da área foliar lesionada.

pla variabilidade entre as linhagens e possibilidades de sucesso com a seleção, dentro de cada sistema de cultivo (Tabela 2).

Na Tabela 3, estão as estimativas das médias das características avaliadas nos 25 genótipos, nos quatro sistemas de cultivo. Verifica-se que a maior produtividade de grãos foi alcançada no sistema de cultivo com irrigação por inundação contínua e semeadura em linhas $\left(8.684 \mathrm{~kg} \mathrm{ha}^{-1}\right)$, que diferiu, significativamente, das médias obtidas nos demais sistemas. Por outro lado, os sistemas de cultivo com irrigação intermitente, independentemente do método de semeadura, apresentaram as menores produtividades de grãos. Estes resultados diferem dos encontrados por Medeiros et al. (1995), que, na comparação de sistemas de irrigação por inundação contínua, inundação intermitente, saturação do solo e combinação destes sistemas, verificaram não haver diferenças significativas entre eles, em relação à produtividade de grãos de arroz.
Por outro lado, Medeiros et al. (2008) observaram maior produtividade de grãos em sistemas de irrigação por inundação contínua. Entre outros fatores, atribuíram isto à eliminação total do estresse hídrico, ao melhor controle de plantas daninhas, ao aumento na disponibilidade de nutrientes e à maior tolerância das plantas a doenças, neste sistema. Pelos dados obtidos no presente trabalho (Tabela 3), verifica-se que o sistema de irrigação por inundação contínua e semeadura em linhas propiciou menor incidência de brusone na folha e de mancha de grãos, em relação à ocorrência observada nos demais sistemas.

Com relação às estimativas das médias para altura de planta e ciclo (Tabela 3), os genótipos avaliados nos sistemas S1 e S2 foram, em média, mais altos e mais precoces do que nos sistemas S3 e S4. Entretanto, estas diferenças não foram consideradas como fator de restrição na seleção das linhagens mais produtivas, pois o intervalo obtido está dentro do recomendado, normalmente, para o arroz irrigado.

Tabela 3. Produção de grãos, altura de planta, ciclo, notas de brusone na folha e mancha de grãos, de genótipos de arroz, em quatro sistemas de cultivo, em várzea do Rio Branco (Cantá, RR, safra 2007/2008).

\begin{tabular}{|c|c|c|c|c|c|}
\hline Sistemas de cultivo & $\begin{array}{l}\text { Produção de grãos } \\
\left.(\mathrm{kg} \mathrm{ha})^{-1}\right)^{1}\end{array}$ & $\begin{array}{l}\text { Altura de planta } \\
(\mathrm{cm})^{1}\end{array}$ & $\begin{array}{l}\text { Ciclo } \\
{\text { (dias })^{1}}^{1}\end{array}$ & $\begin{array}{c}\text { Brusone } \\
\text { na folha } 1,2\end{array}$ & $\begin{array}{c}\text { Mancha } \\
\text { de grãos } 1,2\end{array}$ \\
\hline $\mathrm{S}_{1}$ & $8.684 \mathrm{a}$ & $93 \mathrm{a}$ & $106 \mathrm{c}$ & $1,74 \mathrm{~d}$ & $3,12 \mathrm{c}$ \\
\hline $\mathrm{S}_{2}$ & $8.288 \mathrm{~b}$ & 93 a & $105 \mathrm{c}$ & $2,54 \mathrm{~b}$ & $3,20 \mathrm{c}$ \\
\hline $\mathrm{S}_{3}^{2}$ & $7.516 \mathrm{c}$ & $82 \mathrm{c}$ & $108 \mathrm{~b}$ & $2,86 \mathrm{a}$ & $4,14 \mathrm{a}$ \\
\hline $\mathrm{S}_{4}$ & $7.538 \mathrm{c}$ & $84 \mathrm{~b}$ & $110 \mathrm{a}$ & $2,12 \mathrm{c}$ & $3,70 \mathrm{~b}$ \\
\hline
\end{tabular}

$\mathrm{S}_{1}$ : irrigação contínua e semeadura em linhas; $\mathrm{S}_{2}$ : irrigação contínua e semeadura a lanço; $\mathrm{S}_{3}$ : irrigação intermitente e semeadura em linhas; $\mathrm{S}_{4}$ : irrigação intermitente $\mathrm{e}$ semeadura a lanço.

${ }^{1}$ Médias seguidas da mesma letra, na coluna, pertencem ao mesmo grupo, a 5\% de probabilidade, pelo teste de Scott \& Knott.

${ }^{2}$ Escala de notas visuais de doenças ( 1 a 9), sendo 1 = ausência de lesão e $9=$ mais de $75 \%$ da área foliar lesionada.

${ }^{* * *}$ Valores significativos, a $1 \%$ de probabilidade, pelo teste $\mathrm{F}$. 
Tabela 4. Estimativas do coeficiente de correlação de Pearson (r), obtidas com base na média de características avaliadas em ensaios de Valor de Cultivo e Uso para linhagens de arroz, sob quatro diferentes sistemas de cultivo (Cantá, RR, safra 2007/2008).

\begin{tabular}{ccccc}
\hline \multirow{2}{*}{ Característica } & \multicolumn{4}{c}{ Correlação fenotípica } \\
\cline { 2 - 5 } & $\mathrm{BF}^{1}$ & $\mathrm{MG}^{1}$ & Ciclo & Produção \\
\hline Altura & $-0,08^{\mathrm{ns}}$ & $-0,13^{\mathrm{ns}}$ & $0,03^{\mathrm{ns}}$ & $-0,38^{*}$ \\
BF & - & $0,13^{\mathrm{ns}}$ & $-0,61^{* *}$ & $0,16^{\mathrm{ns}}$ \\
MG & - & - & $-0,26^{\mathrm{ns}}$ & $-0,09^{\mathrm{ns}}$ \\
Ciclo & - & - & - & $-0,18^{\mathrm{ns}}$ \\
\hline
\end{tabular}

${ }^{1} \mathrm{BF}$ : brusone na folha; MG: mancha de grãos.

${ }^{\text {ns }}$ Valores não significativos, pelo teste t-Student, em nível de 5\% de probabilidade.

" $\mathrm{e}^{* *}$ : valores significativos, a $5 \%$ e $1 \%$ de probabilidade, respectivamente, pelo teste t.

As estimativas dos coeficientes de correlação de Pearson (r), entre as características avaliadas, na média de todos os sistemas, são apresentadas na Tabela 4. Houve correlação negativa e significativa entre altura de planta e produção de grãos e entre notas de brusone na folha e ciclo. Isto permite inferir que as linhagens mais altas foram menos produtivas e que as linhagens mais precoces, em geral, apresentaram maiores notas de brusone nas folhas, muito embora estas notas tenham sido baixas e não limitantes à seleção dos genótipos, para os diferentes sistemas. Genótipos de arroz irrigado com maiores alturas de planta, comparados àqueles com plantas mais baixas, em geral, perfilham menos e, consequentemente, produzem menor número de panículas e menor produtividade de grãos. Por outro lado, genótipos de ciclo precoce, com fase vegetativa mais curta, possuem menor capacidade de recuperação sob ocorrência de brusone, se comparados a genótipos de ciclo mais longo. No entanto, Reis et al. (2007) relatam que genótipos de arroz respondem de modo diferenciado ao efeito de ambiente, no tocante à incidência de doenças, tornando esta característica de baixa previsibilidade.

Nas Tabelas 5, 6 e 7, estão os dados referentes às estimativas das médias dos 25 genótipos de arroz irrigado, avaliados nos quatro sistemas de cultivo, para as características altura de planta, ciclo e produtividade de grãos, que foram as que apresentaram diferenças significativas, nas análises de variância conjuntas.

A altura média, para todos os genótipos, com base nos quatro sistemas de cultivo, foi de $88 \mathrm{~cm}$. Na média dos quatro sistemas de cultivo, os genótipos
Tabela 5. Dados médios ${ }^{1}$ de altura de planta $(\mathrm{cm})$ de genótipos de arroz, avaliados em quatro diferentes sistemas de cultivo e com base na média dos quatro sistemas, em várzea do Rio Branco (Cantá, RR, safra 2007/2008).

\begin{tabular}{|c|c|c|c|c|c|}
\hline \multirow[b]{2}{*}{ Genótipos } & \multicolumn{4}{|c|}{ Sistemas de cultivo } & \multirow{2}{*}{ Média } \\
\hline & $\mathrm{S}_{1}$ & $\mathrm{~S}_{2}$ & $\mathrm{~S}_{3}$ & $\mathrm{~S}_{4}$ & \\
\hline IRGA $417^{(t)}$ & $94 \mathrm{Aa}$ & $91 \mathrm{Ab}$ & $83 \mathrm{Ba}$ & $81 \mathrm{Bb}$ & $87 \mathrm{c}$ \\
\hline BRA051250 & $90 \mathrm{Ab}$ & $90 \mathrm{Ab}$ & $80 \mathrm{Bb}$ & $80 \mathrm{Bb}$ & $85 \mathrm{c}$ \\
\hline BRA051126 & $96 \mathrm{Aa}$ & $97 \mathrm{Aa}$ & $88 \mathrm{Ba}$ & $87 \mathrm{Ba}$ & $92 \mathrm{a}$ \\
\hline BRA041049 & $89 \mathrm{Ab}$ & $90 \mathrm{Ab}$ & $80 \mathrm{Bb}$ & $79 \mathrm{Bb}$ & $85 \mathrm{c}$ \\
\hline BRS Jaçanã & $97 \mathrm{Aa}$ & $93 \mathrm{Ab}$ & $79 \mathrm{Cb}$ & $87 \mathrm{Ba}$ & $89 \mathrm{~b}$ \\
\hline BRS Jaburu & $92 \mathrm{Ab}$ & $91 \mathrm{Ab}$ & $78 \mathrm{Bb}$ & $79 \mathrm{Bb}$ & $85 \mathrm{c}$ \\
\hline BRA051129 & $97 \mathrm{Aa}$ & $97 \mathrm{Aa}$ & $83 \mathrm{Ba}$ & $89 \mathrm{Ba}$ & $91 \mathrm{a}$ \\
\hline BRA051083 & $88 \mathrm{Ab}$ & $92 \mathrm{Ab}$ & $81 \mathrm{Bb}$ & $82 \mathrm{Bb}$ & $86 \mathrm{c}$ \\
\hline BRA051103 & $93 \mathrm{Aa}$ & $92 \mathrm{Ab}$ & $78 \mathrm{Bb}$ & $79 \mathrm{Bb}$ & $85 \mathrm{c}$ \\
\hline BRA051130 & $94 \mathrm{Aa}$ & $97 \mathrm{Aa}$ & $87 \mathrm{Ba}$ & $89 \mathrm{Ba}$ & $92 \mathrm{a}$ \\
\hline CNA10892 & $86 \mathrm{Ab}$ & $90 \mathrm{Ab}$ & $75 \mathrm{Bb}$ & $83 \mathrm{Bb}$ & $84 \mathrm{c}$ \\
\hline Roraima $^{(t)}$ & $92 \mathrm{Ab}$ & $92 \mathrm{Ab}$ & $76 \mathrm{Bb}$ & $75 \mathrm{Bb}$ & $84 c$ \\
\hline BRA051252 & $92 \mathrm{Ab}$ & $92 \mathrm{Ab}$ & $76 \mathrm{Bb}$ & $81 \mathrm{Bb}$ & $85 \mathrm{c}$ \\
\hline BRA051125 & $95 \mathrm{Aa}$ & $94 \mathrm{Ab}$ & $83 \mathrm{Ba}$ & $86 \mathrm{Ba}$ & $90 \mathrm{~b}$ \\
\hline BRIRGA409 ${ }^{(t)}$ & $99 \mathrm{Aa}$ & $98 \mathrm{Aa}$ & $88 \mathrm{Ba}$ & $89 \mathrm{Ba}$ & $94 \mathrm{a}$ \\
\hline Metica 1 & $93 \mathrm{Aa}$ & $90 \mathrm{Ab}$ & $88 \mathrm{Aa}$ & $87 \mathrm{Aa}$ & $90 \mathrm{~b}$ \\
\hline BRA051067 & $91 \mathrm{Ab}$ & $89 \mathrm{Ab}$ & $76 \mathrm{Bb}$ & $78 \mathrm{Bb}$ & $84 \mathrm{c}$ \\
\hline CNA110114 & $91 \mathrm{Ab}$ & $91 \mathrm{Ab}$ & $84 \mathrm{Ba}$ & $84 \mathrm{Bb}$ & $87 \mathrm{c}$ \\
\hline BRA051077 & $89 \mathrm{Ab}$ & $91 \mathrm{Ab}$ & $78 \mathrm{Bb}$ & $82 \mathrm{Bb}$ & $85 \mathrm{c}$ \\
\hline BRA051135 & $96 \mathrm{Aa}$ & $93 \mathrm{Ab}$ & $87 \mathrm{Ba}$ & $87 \mathrm{Bb}$ & $91 \mathrm{a}$ \\
\hline CNA10900 & $88 \mathrm{Ab}$ & $90 \mathrm{Ab}$ & $76 \mathrm{Bb}$ & $75 \mathrm{Bb}$ & $82 \mathrm{c}$ \\
\hline BRA051134 & $98 \mathrm{Aa}$ & $93 \mathrm{Ab}$ & $86 \mathrm{Ba}$ & $87 \mathrm{Ba}$ & $91 \mathrm{a}$ \\
\hline BRA051107 & $89 \mathrm{Ab}$ & $95 \mathrm{Aa}$ & $78 \mathrm{Bb}$ & $80 \mathrm{Bb}$ & $86 \mathrm{c}$ \\
\hline BRA051108 & $98 \mathrm{Aa}$ & $94 \mathrm{Ab}$ & $85 \mathrm{Ba}$ & $93 \mathrm{Ba}$ & $92 \mathrm{a}$ \\
\hline BRA051179 & $95 \mathrm{Aa}$ & $100 \mathrm{Aa}$ & $88 \mathrm{Ba}$ & $95 \mathrm{Aa}$ & $95 \mathrm{a}$ \\
\hline Média & $93 \mathrm{~A}$ & $93 \mathrm{~A}$ & $82 \mathrm{C}$ & $84 \mathrm{~B}$ & 88 \\
\hline Mínimo & 86 & 89 & 75 & 75 & \\
\hline Ma & 99 & 100 & 88 & 95 & \\
\hline
\end{tabular}

$\mathrm{S}_{1}$ : irrigação contínua e semeadura em linhas; $\mathrm{S}_{2}$ : irrigação contínua e semeadura a lanço; $\mathrm{S}_{3}$ : irrigação intermitente e semeadura em linhas; $\mathrm{S}_{4}$ : irrigação intermitente e semeadura a lanço; ${ }^{(\mathrm{t})}$ : testemunha.

Médias seguidas da mesma letra minúscula, na coluna, e maiúscula, na linha, pertencem ao mesmo grupo, a $5 \%$ de probabilidade, pelo teste de Scott \& Knott.

mais altos foram BRA $051179(95 \mathrm{~cm})$, BR IRGA 409 $(94 \mathrm{~cm})$, BRA $051126(92 \mathrm{~cm})$, BRA $051130(92 \mathrm{~cm})$, BRA $051108(92 \mathrm{~cm})$, BRA $051129(91 \mathrm{~cm})$ e BRA $051135(91 \mathrm{~cm})$. Dentre estes, apenas as linhagens BRA 051135, BRA 051126 e BRA 051108 estiveram entre as mais produtivas, corroborando a correlação negativa e significativa comentada anteriormente, entre altura de planta e produtividade (Tabela 4). No entanto, para arroz irrigado, Fagundes et al. (2007) citam que a altura adequada para o cultivo mecanizado é de $80-90 \mathrm{~cm}$, valor semelhante às médias obtidas neste trabalho.

Os genótipos apresentaram ciclo (emergência à colheita) com média de 107 dias, sendo os mais precoces com 101 dias e os mais tardios com 114 dias. As cultivares testemunhas IRGA 417, Roraima e BR IRGA 409 apresentaram ciclos semelhantes, em torno de 103 dias. A cultivar de ciclo mais tardio, na 
Tabela 6. Dados médios ${ }^{1}$ de ciclo da planta (dias até a maturação completa), em quatro sistemas de cultivo de arroz e com base na média dos quatro sistemas, em várzea do Rio Branco (Cantá, RR, safra 2007/2008).

\begin{tabular}{lccccc}
\hline \multirow{2}{*}{ Genótipos } & \multicolumn{5}{c}{ Sistemas de cultivo } \\
\cline { 2 - 5 } & $\mathrm{S}_{1}$ & $\mathrm{~S}_{2}$ & $\mathrm{~S}_{3}$ & $\mathrm{~S}_{4}$ & Média \\
\hline IRGA 417 & $103 \mathrm{Ab}$ & $101 \mathrm{Ad}$ & $101 \mathrm{Ab}$ & $102 \mathrm{Ad}$ & $102 \mathrm{~d}$ \\
BRA051250 & $108 \mathrm{Ba}$ & $106 \mathrm{Bc}$ & $106 \mathrm{Bb}$ & $111 \mathrm{Ab}$ & $107 \mathrm{~b}$ \\
BRA051126 & $104 \mathrm{Bb}$ & $105 \mathrm{Bc}$ & $111 \mathrm{Aa}$ & $110 \mathrm{Ab}$ & $108 \mathrm{~b}$ \\
BRA041049 & $105 \mathrm{Ab}$ & $102 \mathrm{Bd}$ & $107 \mathrm{Ab}$ & $106 \mathrm{Ac}$ & $105 \mathrm{c}$ \\
BRS Jaçanã & $105 \mathrm{Ab}$ & $101 \mathrm{Bd}$ & $103 \mathrm{Bb}$ & $107 \mathrm{Ac}$ & $104 \mathrm{c}$ \\
BRS Jaburu & $108 \mathrm{Ba}$ & $104 \mathrm{Cc}$ & $109 \mathrm{Ba}$ & $113 \mathrm{Aa}$ & $108 \mathrm{~b}$ \\
BRA051129 & $105 \mathrm{Ab}$ & $102 \mathrm{Ad}$ & $105 \mathrm{Ab}$ & $105 \mathrm{Ac}$ & $104 \mathrm{c}$ \\
BRA051083 & $107 \mathrm{Ba}$ & $104 \mathrm{Bc}$ & $104 \mathrm{Bb}$ & $112 \mathrm{Aa}$ & $107 \mathrm{~b}$ \\
BRA051103 & $106 \mathrm{Aa}$ & $104 \mathrm{Ac}$ & $105 \mathrm{Ab}$ & $108 \mathrm{Ac}$ & $106 \mathrm{c}$ \\
BRA051130 & $107 \mathrm{Ba}$ & $104 \mathrm{Bc}$ & $111 \mathrm{Aa}$ & $113 \mathrm{Aa}$ & $109 \mathrm{~b}$ \\
CNA10892 & $102 \mathrm{Cb}$ & $111 \mathrm{Ba}$ & $109 \mathrm{Ba}$ & $114 \mathrm{Aa}$ & $109 \mathrm{~b}$ \\
Roraima ${ }^{(t)}$ & $102 \mathrm{Bb}$ & $101 \mathrm{Bd}$ & $105 \mathrm{Bb}$ & $110 \mathrm{Ab}$ & $104 \mathrm{c}$ \\
BRA051252 & $105 \mathrm{Bb}$ & $106 \mathrm{Bc}$ & $109 \mathrm{Aa}$ & $110 \mathrm{Ab}$ & $107 \mathrm{~b}$ \\
BRA051125 & $104 \mathrm{Bb}$ & $104 \mathrm{Bc}$ & $105 \mathrm{Bb}$ & $110 \mathrm{Ab}$ & $106 \mathrm{c}$ \\
BRIRGA409 & $102 \mathrm{Bb}$ & $102 \mathrm{Bd}$ & $105 \mathrm{Bb}$ & $110 \mathrm{Ab}$ & $105 \mathrm{c}$ \\
Metica 1 & $110 \mathrm{Aa}$ & $113 \mathrm{Aa}$ & $112 \mathrm{Aa}$ & $113 \mathrm{Aa}$ & $112 \mathrm{a}$ \\
BRA051067 & $108 \mathrm{Ba}$ & $104 \mathrm{Bc}$ & $110 \mathrm{Aa}$ & $110 \mathrm{Ab}$ & $108 \mathrm{~b}$ \\
CNA110114 & $103 \mathrm{Bb}$ & $106 \mathrm{Bc}$ & $112 \mathrm{Aa}$ & $113 \mathrm{Aa}$ & $108 \mathrm{~b}$ \\
BRA051077 & $106 \mathrm{Ba}$ & $104 \mathrm{Bc}$ & $111 \mathrm{Aa}$ & $114 \mathrm{Aa}$ & $109 \mathrm{~b}$ \\
BRA051135 & $106 \mathrm{Aa}$ & $109 \mathrm{Ab}$ & $110 \mathrm{Aa}$ & $111 \mathrm{Ab}$ & $109 \mathrm{~b}$ \\
CNA10900 & $106 \mathrm{Ba}$ & $107 \mathrm{Bb}$ & $111 \mathrm{Aa}$ & $113 \mathrm{Aa}$ & $109 \mathrm{~b}$ \\
BRA051134 & $106 \mathrm{Aa}$ & $107 \mathrm{Ab}$ & $110 \mathrm{Aa}$ & $110 \mathrm{Ab}$ & $108 \mathrm{~b}$ \\
BRA051107 & $104 \mathrm{Bb}$ & $104 \mathrm{Bc}$ & $107 \mathrm{Ab}$ & $109 \mathrm{Ab}$ & $106 \mathrm{c}$ \\
BRA051108 & $108 \mathrm{Ba}$ & $108 \mathrm{Bb}$ & $110 \mathrm{Ba}$ & $112 \mathrm{Aa}$ & $109 \mathrm{~b}$ \\
BRA051179 & $107 \mathrm{Aa}$ & $107 \mathrm{Ab}$ & $108 \mathrm{Aa}$ & $110 \mathrm{Ab}$ & $108 \mathrm{~b}$ \\
\hline Média & $106 \mathrm{C}$ & $105 \mathrm{C}$ & $108 \mathrm{~B}$ & $110 \mathrm{~A}$ & 107 \\
Mínimo & 102 & 101 & 101 & 102 & \\
Máximo & 110 & 113 & 112 & 114 & \\
\hline CV $=2,38 \%$ & & & & & \\
\hline
\end{tabular}

$\mathrm{CV}=2,38 \%$

$\mathrm{S}_{1}$ : irrigação contínua e semeadura em linhas; $\mathrm{S}_{2}$ : irrigação contínua e semeadura a lanço; $\mathrm{S}_{3}$ : irrigação intermitente e semeadura em linhas; $\mathrm{S}_{4}$ : irrigação intermitente e semeadura a lanço; ${ }^{(t)}$ : testemunha.

${ }^{1}$ Médias seguidas da mesma letra minúscula, na coluna, e maiúscula, na linha, pertencem ao mesmo grupo, a $5 \%$ de probabilidade, pelo teste de Scott \& Knott.

média dos quatro sistemas, foi Metica 1 (112 dias). De modo geral, os genótipos apresentaram ciclo mais tardio, quando manejados com irrigação intermitente (Tabela 6). Resultados semelhantes foram obtidos por Cordeiro (2005a), que observou maior número de dias para floração, em linhagens avaliadas sob sistema de várzea úmida e semeio em linhas.

Segundo Gomes et al. (1999), a manutenção de lâmina de água sobre a superfície do solo, condição normalmente observada em culturas de arroz irrigado por inundação contínua, exerce uma série de influências, tanto nas plantas de arroz como no solo. Estes efeitos estão relacionados, entre outros, a aspectos fisiológicos das plantas, bem como ao controle de plantas daninhas e algumas pragas e doenças. No presente trabalho, a irrigação intermitente pode ter favorecido uma maior competição das plantas de arroz com as plantas daninhas, além de ter propiciado
Tabela 7. Produtividade média ${ }^{1}$ de grãos $\left(\mathrm{kg} \mathrm{ha}^{-1}\right)$, em quatro sistemas de cultivo de arroz e com base na média dos sistemas, em várzea do Rio Branco (Cantá, RR, safra 2007/2008).

\begin{tabular}{|c|c|c|c|c|c|}
\hline \multirow{2}{*}{ Genótipo } & \multicolumn{4}{|c|}{ Sistemas de Cultivo } & \multirow{2}{*}{ Média } \\
\hline & $S_{1}$ & $\mathrm{~S}_{2}$ & $\mathrm{~S}_{3}$ & $\mathrm{~S}_{4}$ & \\
\hline$\overline{\text { IRGA } 417^{(t)}}$ & $9.590 \mathrm{Aa}$ & $9.362 \mathrm{Aa}$ & $8.298 \mathrm{Ba}$ & $8.479 \mathrm{Ba}$ & $8.932 \mathrm{a}$ \\
\hline BRA051250 & $9.529 \mathrm{Aa}$ & $9.509 \mathrm{Aa}$ & $7.225 \mathrm{Cb}$ & $8.400 \mathrm{Ba}$ & $8.666 \mathrm{a}$ \\
\hline RA051126 & $9.363 \mathrm{Aa}$ & $7.638 \mathrm{Bb}$ & $7.537 \mathrm{Ba}$ & $8.114 \mathrm{Ba}$ & \\
\hline BRA041049 & $9.306 \mathrm{Aa}$ & $8.508 \mathrm{Bb}$ & $7.740 \mathrm{Ba}$ & $6.355 \mathrm{Cc}$ & $77 \mathrm{c}$ \\
\hline BRS Jaçanã & $9.147 \mathrm{Aa}$ & $8.987 \mathrm{Aa}$ & $7.530 \mathrm{Bb}$ & $7.365 \mathrm{Bb}$ & $7 \mathrm{~b}$ \\
\hline BRS Jaburu & $9.066 \mathrm{Aa}$ & $7.851 \mathrm{Bb}$ & $7.544 \mathrm{Bb}$ & $8.309 \mathrm{Ba}$ & $8.193 \mathrm{~b}$ \\
\hline 3RA051129 & $9.055 \mathrm{Aa}$ & $7.705 \mathrm{Bb}$ & $7.382 \mathrm{Bb}$ & $7.571 \mathrm{Ba}$ & $7.928 \mathrm{c}$ \\
\hline RA051083 & $62 \mathrm{Aa}$ & $8.698 \mathrm{Aa}$ & $7.012 \mathrm{Bb}$ & $6.938 \mathrm{Bb}$ & $02 \mathrm{c}$ \\
\hline BRA0 & $38 \mathrm{Aa}$ & $7.927 \mathrm{Bb}$ & $7.430 \mathrm{Bb}$ & $7.873 \mathrm{Ba}$ & $2 \mathrm{c}$ \\
\hline BRA051130 & $8.910 \mathrm{Aa}$ & $8.464 \mathrm{Ab}$ & $6.948 \mathrm{Bb}$ & $6.818 \mathrm{Bb}$ & $7.785 \mathrm{c}$ \\
\hline CNA10892 & $8.845 \mathrm{Aa}$ & $9.048 \mathrm{Aa}$ & $7.841 \mathrm{Ba}$ & $6.365 \mathrm{Cc}$ & $25 \mathrm{c}$ \\
\hline Roraima $^{(t)}$ & $8.795 \mathrm{Aa}$ & $8.062 \mathrm{Ab}$ & $7.332 \mathrm{Bb}$ & $7.096 \mathrm{Bb}$ & $7.821 \mathrm{c}$ \\
\hline $\mathrm{BRA}$ & $777 \mathrm{Aa}$ & $8.424 \mathrm{Ab}$ & $7.243 \mathrm{Bb}$ & $7.424 \mathrm{Bb}$ & $67 \mathrm{c}$ \\
\hline BRA051125 & $8.764 \mathrm{Aa}$ & $7.615 \mathrm{Bb}$ & $7.409 \mathrm{Bb}$ & $8.025 \mathrm{Ba}$ & $7.953 \mathrm{c}$ \\
\hline BRIRGA409(t) & $8.738 \mathrm{Aa}$ & $8.072 \mathrm{Ab}$ & $6.555 \mathrm{Bb}$ & $6.103 \mathrm{Bc}$ & $7.367 \mathrm{~d}$ \\
\hline Metica 1 & $8.722 \mathrm{Aa}$ & $7.942 \mathrm{Ab}$ & $7.349 \mathrm{Bb}$ & $7.002 \mathrm{Bb}$ & $7.754 \mathrm{c}$ \\
\hline 067 & $710 \mathrm{Aa}$ & $8.015 \mathrm{Bb}$ & $7.145 \mathrm{Bb}$ & $7.716 \mathrm{Ba}$ & $7.897 \mathrm{c}$ \\
\hline 4 & $8.540 \mathrm{Aa}$ & $7.567 \mathrm{Bb}$ & $7.818 \mathrm{Ba}$ & $8.752 \mathrm{Aa}$ & $8.169 \mathrm{~b}$ \\
\hline 17 & $8.519 \mathrm{Aa}$ & $7.961 \mathrm{Ab}$ & $8.115 \mathrm{Aa}$ & $8.007 \mathrm{Aa}$ & $8.151 \mathrm{~b}$ \\
\hline 35 & $18 \mathrm{Aa}$ & $8.121 \mathrm{Ab}$ & $9.114 \mathrm{Aa}$ & 7.215 Bb & $7 \mathrm{~b}$ \\
\hline & $9 \mathrm{Bb}$ & $9.223 \mathrm{Aa}$ & $7.367 \mathrm{Bb}$ & $0 \mathrm{Ba}$ & \\
\hline & $2 \mathrm{Ab}$ & $8 \mathrm{Ab}$ & $0 \mathrm{Aa}$ & $\mathrm{Aa}$ & \\
\hline & $1 \mathrm{Ab}$ & $9 \mathrm{Ab}$ & $6.932 \mathrm{Ab}$ & $6 \mathrm{Aa}$ & \\
\hline & $7.616 \mathrm{Ab}$ & $3 \mathrm{Ab}$ & $8.049 \mathrm{Aa}$ & $5 \mathrm{Aa}$ & \\
\hline BRA051179 & $6.632 \mathrm{Bc}$ & $8.124 \mathrm{Ab}$ & $7.133 \mathrm{Bb}$ & $5.950 \mathrm{Bc}$ & $6.960 \mathrm{~d}$ \\
\hline Média & $8.684 \mathrm{~A}$ & $8.288 \mathrm{~B}$ & $7.516 \mathrm{C}$ & $7.538 \mathrm{C}$ & 8.006 \\
\hline Mínimo & 6.632 & 7.567 & 6.555 & 5.950 & \\
\hline Máximo & 9.590 & 9.509 & 9.114 & 8.752 & \\
\hline \multicolumn{6}{|l|}{$\mathrm{CV}=8,80 \%$} \\
\hline \multicolumn{6}{|c|}{$\begin{array}{l}\mathrm{S}_{1} \text { : irrigação contínua e semeadura em linhas; } \mathrm{S}_{2} \text { : irrigação contínua e semeadura a } \\
\text { lanço; } \mathrm{S}_{3} \text { : irrigação intermitente e semeadura em linhas; } \mathrm{S}_{4} \text { : irrigação intermitente } \\
\text { e semeadura a lanço; }{ }^{(t)} \text { : testemunha. } \\
{ }^{1} \text { Médias seguidas da mesma letra minúscula, na coluna, e maiúscula, na linha, } \\
\text { pertencem ao mesmo grupo, a } 5 \% \text { de probabilidade, pelo teste de Scott \& } \\
\text { Knott. }\end{array}$} \\
\hline
\end{tabular}

maior ocorrência de doenças, aumentado, assim, o ciclo da planta.

Deve-se considerar, também, que a ação física da lâmina de água pode servir como efeito termorregulador, ou seja, na presença de estresse, a planta completa seu ciclo em período mais longo. Segundo Stone et al. (1990), os efeitos do estresse hídrico no arroz irrigado podem ocorrer quando o conteúdo da água do solo cai abaixo da saturação. Isto ocorreu nos sistemas com irrigação intermitente, em função da oscilação da umidade do solo entre a saturação completa e a capacidade de campo. Entretanto, como não houve correlação entre ciclo e produtividade de grãos (Tabela 4), admite-se que os ciclos obtidos pelos diferentes genótipos não foram fator de limitação para a seleção das linhagens mais produtivas.

Com relação à produtividade de grãos, verificaram-se valores bastante satisfatórios, com média geral de $8.006 \mathrm{~kg} \mathrm{ha}^{-1}$ (Tabela 7). A menor produtividade obtida foi $5.950 \mathrm{~kg} \mathrm{ha}^{-1} \mathrm{e}$ a maior $9.590 \mathrm{~kg} \mathrm{ha}^{-1}$. Dentro de cada sistema, verifica-se que a cultivar 
IRGA 417 foi uma das mais produtivas, com bom desempenho, nas diferentes condições. As cultivares BRS Jaçanã, BRS Jaburu e Roraima, embora com menores produtividades, em relação à IRGA 417, também apresentaram boa adaptação aos diferentes sistemas. Resultado semelhante foi observado por Martini et al. (2009), avaliando os sistemas de manejo de irrigação contínua e intermitente, para a cultivar IRGA 422 CL. Os autores obtiveram produtividades, na média dos dois sistemas, em torno de $9.000 \mathrm{~kg} \mathrm{ha}^{-1}$, o que mostra a boa adaptação da cultivar aos sistemas testados.

Entre as linhagens experimentais avaliadas na presente pesquisa, destacaram-se, como promissoras, a BRA 051250 (9.529 $\left.\mathrm{kg} \mathrm{ha}^{-1}\right)$ e BRA 051126 (9.363 kg ha-1), para S1; BRA 051250 $\left(9.509 \mathrm{~kg} \mathrm{ha}^{-1}\right)$ e CNA $10900\left(9.223 \mathrm{~kg} \mathrm{ha}^{-1}\right)$, para S2; BRA 051135 (9.114 kg ha-1), para S3; e CNA 110114 $\left(8.752 \mathrm{~kg} \mathrm{ha}^{-1}\right)$, para S4. Estas foram superiores em $19 \%, 16,9 \%, 18,7 \%, 15,2 \%, 13,8 \%$ e $9,3 \%$, respectivamente, à média geral de todos os genótipos, nos diferentes sistemas $\left(8.006 \mathrm{~kg} \mathrm{ha}^{-1}\right)$.

Cordeiro (2005a, 2005b), avaliando novas linhagens de arroz, em três sistemas de cultivo em várzea de Roraima (irrigação contínua e semeio a lanço, irrigação contínua e semeio em linhas e várzea úmida e semeio em linhas), obteve produtividades, na média dos três sistemas, variando de $6.538 \mathrm{~kg} \mathrm{ha}^{-1} \mathrm{a}$ $8.234 \mathrm{~kg} \mathrm{ha}^{-1}$. Observando-se a produtividade média obtida no presente estudo (8.006 kg ha $\left.\mathrm{k}^{-1}\right)$, com máxima de $9.590 \mathrm{~kg} \mathrm{ha}^{-1}$, pode-se inferir, portanto, que as novas linhagens desenvolvidas nos programas de melhoramento genético têm apresentado maiores produtividades, independentemente dos sistemas de cultivo utilizados. Estas produtividades são semelhantes àquelas obtidas em regiões de cultivo tradicional de arroz irrigado, como o Estado do Rio Grande do Sul. Exemplo disto é a pesquisa realizada por Fagundes et al. (2007), avaliando nove cultivares desenvolvidas pela Embrapa, em seis ambientes, em que obtiveram produtividades médias entre $6.619 \mathrm{~kg} \mathrm{ha}^{-1} \mathrm{e}$ $10.100 \mathrm{~kg} \mathrm{ha}^{-1}$. Isto revela o potencial adaptativo dos genótipos avaliados na presente pesquisa.

\section{CONCLUSÕES}

1. Nas condições avaliadas, o sistema de cultivo do arroz com irrigação por inundação contínua e semeadura em linhas resulta em maior produtividade de grãos, enquanto os sistemas com irrigação intermitente, independentemente do método de semeadura, são menos produtivos.

2. As cultivares IRGA 417, BRS Jaçanã, BRS Jaburu e Roraima apresentaram produções superiores, nos diferentes sistemas de cultivo avaliados.

3. As linhagens experimentais BRA 051250, BRA 051126, CNA 10900, BRA 051135 e CNA 110114 são as mais promissoras para liberações futuras de cultivares adaptadas aos sistemas de produção local.

\section{REFERÊNCIAS}

AZAMBUJA, I. H. V.; VERNETTI JÚNIOR, F. J.; MAGALHÃES JÚNIOR, A. M. Aspectos sócioeconômicos da produção do arroz. In: GOMES, A. S.; MAGALHÃES JÚNIOR, A. M. Arroz irrigado no Sul do Brasil. Brasília, DF: Embrapa Informação Tecnológica, 2004. p. 23-44.

CORDEIRO, A. C. C. Ensaios de valor de cultivo e uso de arroz irrigado de ciclo precoce em várzea de Roraima, safra 2003/2004. In: CONGRESSO BRASILEIRO DE ARROZ IRRIGADO, 4.; REUNIÃO DA CULTURA DO ARROZ IRRIGADO, 26., 2005, Santa Maria. Anais... Santa Maria: Orium, 2005a. p. 159-161.

CORDEIRO, A. C. C. O cultivo do arroz irrigado em Roraima. In: BARBOSA, R. I. et al. Savanas de Roraima: etnoecologia, biodiversidade e potencialidades agrossilvipastoris. Boa Vista: Femact-RR, 2005b.

CORDEIRO, A. C. C.; MOURÃO JÚNIOR, M. C.; MEDEIROS, R. D. Análise do agronegócio do arroz irrigado em Roraima: período 1981 a 2007. In: CONGRESSO BRASILEIRO DE ARROZ IRRIGADO, 6.; REUNIÃO DA CULTURA DO ARROZ IRRIGADO, 28., 2007, Porto Alegre. Anais... Porto Alegre: Orium, 2007. p. 719-721.

CORDEIRO, A. C. C. et al. O cultivo do arroz irrigado em Roraima. Boa Vista: Embrapa Roraima, 2004. (Documentos, 1).

DUARTE, F. M. Perdas de nitrogênio por volatilização de amônia e eficiência da adubação nitrogenada no cultivo do arroz irrigado. 2006. 85 f. Dissertação (Mestrado em Ciência do Solo)-Centro de Ciências Rurais, Universidade Federal de Santa Maria, Santa Maria, 2006.

EMPRESA BRASILEIRA DE PESQUISA AGROPECUÁRIA (Embrapa). Centro Nacional de Pesquisa de Solos. Sistema brasileiro de classificação de solos. 2. ed. Rio de Janeiro: Embrapa Solos, 2006.

EMPRESA BRASILEIRA DE PESQUISA AGROPECUÁRIA (Embrapa). Serviço Nacional de Levantamento e Conservação de Solos. Manual de métodos de análise de solo. 2. ed. Rio de Janeiro: EmbrapaCNPS, 1997. 
FAGUNDES, P. R. R. et al. Avaliação de cultivares recomendadas de arroz irrigado da Embrapa, no Rio Grande do Sul, 2006/07. In: CONGRESSO BRASILEIRO DE ARROZ IRRIGADO, 6.; REUNIÃO DA CULTURA DO ARROZ IRRIGADO, 28., 2007, Porto Alegre. Anais... Porto Alegre: Orium, 2007. p. 35-37.

FERREIRA, D. F. Sisvar: um programa para análises e ensino de estatística. Symposium, Lavras, v. 6, n. 1, p. 3641, 2008.

GOMES, F. P. Curso de estatística experimental. 11. ed. Piracicaba: Nobel, 1990.

GOMES, A. da S. et al. Manejo da água em arroz irrigado: implicações e recomendações técnicas. In: GOMES, A. de S.; PAULETTO, E. A (Orgs.). Manejo de solo e da água em áreas de várzea. Pelotas: Embrapa Clima Temperado, 1999. p. 163-200.

INTERNATIONAL RICE RESEARCH INSTITUTE (IRRI). Standard evaluation system for rice. Manilla: Inger/Genetic Researches Center, 1996.

MARTINI, L. F. D. et al. Irrigação intermitente permite redução do volume de água aplicado sem afetar a produtividade do arroz irrigado. In: CONGRESSO BRASILEIRO DE ARROZ IRRIGADO, 6., 2009, Porto Alegre. Anais... Porto Alegre: Palotti, 2009. p. 227-230.

MEdeIROS, R. D. de; CORDEIRO, A. C. C.; BENDAHAN, A. B. Irrigação e manejo da água para a cultura do arroz irrigado em Roraima. Boa Vista: Embrapa Roraima, 2008. (Circular técnica, 4).
MEDEIROS, R. D. de; HOLANDA, J. S. de; COSTA, M. C. Manejo de água em arroz irrigado no Estado de Roraima. Lavoura Arrozeira, Porto Alegre, v. 48, n. 1, p. 12-14, 1995.

MEDEIROS, R. D. de et al. Efeito do manejo da água e de herbicidas na cultura do arroz (Oryza sativa, L.) irrigado. Irriga, Botucatu, v. 2, n. 1, p. 38-49, 1997.

PRODUTORES DE VINHOS ALVARINHOS DE MONÇ̃̃O (Provam). Caracterização dos solos, avaliação da aptidão agrícola das terras e indicativo de culturas para as várzeas do Cerrado do Estado de Roraima: relatório final. Belém: Sudam/OEA/Embrapa-CPATU, 1996.

RANGEL, P. H. N. Eficiência da seleção simultânea de arroz (Oryza sativa L.) em várzea, com e sem irrigação controlada. 1990. 128 f. Tese (Doutorado em Genética e Melhoramento de Plantas)-Escola Superior de Agricultura Luiz de Queiroz, Universidade de são Paulo, Piracicaba, 1990.

REIS, M. de S. et al. Comportamento de genótipos de arroz de terras altas sob sistemas de plantio direto e convencional. Pesquisa Agropecuária Tropical, Goiânia, v. 37, n. 4. p. 227-232, out./dez. 2007.

STONE, L. F.; MOREIRA, J. A. A.; SILVEIRA FILHO, A. Manejo de água na cultura do arroz: consumo, ocorrência de plantas daninhas, absorção de nutrientes e características produtivas. Pesquisa Agropecuária Brasileira, Brasília, DF, v. 25, n. 3, p. 323-327, mar. 1990.

ZIMMERMJANN, F. J. P. Estatística aplicada à pesquisa agrícola. Santo Antônio de Goiás: Embrapa Arroz e Feijão, 2004. 